\title{
Constant activation of the RAF-MEK-ERK pathway as a diagnostic and therapeutic target in hairy cell leukemia
}

Enrico Tiacci, ${ }^{1}$ Gianluca Schiavoni, ${ }^{1}$ Maria Paola Martelli, ${ }^{1}$ Emanuela Boveri, ${ }^{2}$ Roberta Pacini, ${ }^{1}$ Alessia Tabarrini Silvia Zibellini, ${ }^{3}$ Alessia Santi, ${ }^{1}$ Valentina Pettirossi, ${ }^{1}$ Elisabetta Fortini, ${ }^{1}$ Stefano Ascani, ${ }^{4}$ Luca Arcaini, ${ }^{3}$ Giorgio Inghirami, ${ }^{5}$ Marco Paulli, ${ }^{2}$ and Brunangelo Falini ${ }^{1}$

${ }^{1}$ Institute of Hematology, Ospedale S. Maria della Misericordia, University of Perugia, Perugia; ${ }^{2}$ Anatomic Pathology Section and ${ }^{3}$ Department of Hematology-Oncology, Fondazione IRCCS Policlinico San Matteo, University of Pavia, Pavia; ${ }^{4}$ Institute of Pathology, Ospedale di Terni, University of Perugia, Perugia; and ${ }^{5}$ Department of Pathology, Center for Experimental Research and Medical Studies, University of Turin, Turin, Italy

The BRAF-V600E mutation defines genetically hairy cell leukemia among B-cell leukemias and lymphomas. In solid tumors, BRAF-V600E is known to aberrantly activate the oncogenic MEK-ERK pathway, and targeted BRAF and/or MEK inhibitors have shown remarkable efficacy in clinical trials in melanoma patients. However, the MEKERK pathway status in hairy cell leukemia has not been thoroughly investigated. We assessed phospho-ERK expression in 37 patients with hairy cell leukemia and 44 patients with neoplasms mimicking hairy cell leukemia (40 splenic marginal zone lymphoma, 2 hairy cell leukemia-variant and 2 splenic lymphoma/leukemia unclassifiable) using immunohistochemistry on routine biopsies and/or Western blotting on purified leukemic cells, and correlated the phospho-ERK status with the BRAF-V600E mutation status. Besides confirming the constant presence of BRAF-V600E in all patients with hairy cell leukemia, we observed ubiquitous phospho-ERK expression in this malignancy. Conversely, all 44 cases with neoplasms mimicking hairy cell leukemia were devoid of BRAF-V600E and none expressed phospho-ERK. Furthermore, the two exceptionally rare cases of non-hairy cell leukemia unclassifiable chronic B-cell neoplasms previously reported to be BRAF-V600 $\mathrm{E}^{+}$on allele-specific polymerase chain reaction lacked phospho-ERK expression as well, suggesting the presence of the mutation in only a small part of the leukemic clone in these cases. In conclusion, our findings support the use of phospho-ERK immunohistochemistry in the differential diagnosis between hairy cell leukemia and its mimics, and establish the MEK-ERK pathway as a rational therapeutic target in this malignancy.

\section{Introduction}

Hairy cell leukemia (HCL) is a distinct entity ${ }^{1}$ exhibiting a peculiar morphology, immunophenotype and gene expression profile, ${ }^{2,3}$ as well as a high sensitivity to purine analogs. ${ }^{4}$ Recently, we identified the BRAF-V600E mutation as the disease-defining genetic event in $\mathrm{HCL},{ }^{5}$ being present and stable during the disease course in all HCL patients and being absent in other B-cell leukemias/lymphomas. ${ }^{5,6}$ Our findings have been subsequently confirmed by others. ${ }^{7-11}$

BRAF is a kinase of the RAS-RAF-MEK-ERK pathway which plays a pivotal role in regulating cell proliferation and survival. ${ }^{12}$ The V600E phospho-mimetic substitution leads to constitutive activation of BRAF that, in turn, phosphorylates MEK (its immediate downstream target) and ERK (the kinase phosphorylated by phospo-MEK). ${ }^{13,14}$ Activation of this pathway appears crucial for HCL cell survival in vitro ${ }^{15}$ and its targeting by BRAF and/or MEK inhibitors (recently shown to be remarkably active in patients with BRAF-V600E $\mathrm{E}^{+}$metastatic melanoma ${ }^{16-18}$ ) would provide new therapeutic opportunities in HCL. However, activation of the MEK-ERK pathway has so far been documented in only a few HCL patients. ${ }^{5,15}$

Our main aim was to evaluate, in a series of $37 \mathrm{HCL}$ patients, the correlation between BRAF-V600E and ERK phosphorylation as a read-out of RAF-MEK-ERK pathway activation. This is a pre-requisite for a successful use of BRAF and/or MEK inhibitors ${ }^{16-18}$ in HCL. As a second aim we assessed whether phospho-ERK ( $p E R K$ ) immunostaining of routine biopsies may improve the differential diagnosis between HCL and HCL-like disorders.

\section{Design and Methods}

\section{Patients}

We investigated 37 patients with HCL, 44 patients with HCL-like leukemias/lymphomas 40 splenic marginal zone lymphomas (SMZL), 2 HCL-variant, 2 splenic leukemia/lymphoma unclassifiable] and 7 patients with CD5-negative B-cell lymphoproliferative disorders not otherwise specifiable (BLPD-NOS). Cases were classified according to the 2008-World Health Organization classification of lymphoid neoplasms. ${ }^{1}$ Patients gave informed consent to analysis of their samples.

\section{BRAF-V600E analysis}

Fifty-one patients (14 HCL, 6 BLPD-NOS, 2 splenic leukemia/lymphoma unclassifiable, $29 \mathrm{SMZL}$ ) had already been characterized for 
the presence of the BRAF-V600E mutation..$^{5-7}$ For 36 newly analyzed patients (23 HCL, 1 BLPD-NOS, 11 SMZL, 1 HCL-variant), genomic DNA was extracted from unfractionated peripheral blood samples (17 HCL) using the OIAamp DNA blood Midi kit (Qiagen), or from MACS-purified blood leukemic cells (6 HCL, 1 BLPD-NOS, 1 SMZL, 1 HCL-variant), post-Ficoll blood mononuclear cells (8 SMZL) and fresh splenectomy specimens (2 SMZL) using the Puregene core kit A (Qiagen). In 11 (9 SMZL and 2 BLPD-NOS) of the 36 newly characterized patients, due to the scarcity of the available genomic DNA, we subjected $10 \mathrm{ng}$ of genomic DNA to whole-genome amplification in duplicate (Illustra GenomiPhi HY DNA Amplification kit, GE Healthcare) before performing the allele-specific polymerase chain reaction (PCR) for BRAF-V600E, and the results obtained (presence or absence of the mutant band) were always concordant in the two whole-genome amplification-DNA replicates.

\section{Immunohistochemistry}

Paraffin sections from fixed/decalcified bone marrow biopsies and from fixed splenectomy specimens (or a fixed lymph node biopsy in a single SMZL case also investigated in the bone marrow) were subjected to antigen retrieval $\left(5 \mathrm{~min}\right.$ at $85^{\circ} \mathrm{C}$ or $\left.97^{\circ} \mathrm{C}\right)$ using Dako-Target Retrieval Solution/High-pH (cat. K8004) in Dako-PT-link (cat. PT101). Sections were then immunostained at room temperature with antibodies against $\mathrm{pERK} 1 / 2$ (rabbit clone D13.14.4E, Cell Signaling Technology, cat. 8201; dilution 1:200, incubation for $30 \mathrm{~min}$ ) or CD20 (mouse clone L26, Dako, cat. $\mathrm{M} 0755$; dilution 1:500, incubation for $20 \mathrm{~min}$ ), followed by detection with the Dako-REAL LSAB+ system (cat. K5005) and hematoxylin counterstaining for $5 \mathrm{~min}$. To assess the specificity of pERK staining, paraffin sections were also immunostained after preincubating the anti-pERK1/2 antibody with its blocking phosphopeptide (Cell Signaling Technologies, cat. \#1150).

Double immuno-enzymatic staining with the anti-pERK1/2 antibody and the anti-PAX5 antibody (mouse clone 29, BD Biosciences, cat. 610863; 1:50 dilution, incubation for $30 \mathrm{~min}$ ) was performed in $21 \mathrm{HCL}$ biopsies, using an immuno-peroxidase detection system (Dako LSAB+ HRP, cat. K0690; Dako liquid $\mathrm{DAB}+$ Substrate and Chromogen, cat. K3468) for the anti-PAX5 antibody, followed by an immuno-alkaline phosphatase detection system (Dako REAL LSAB+, cat. K5005, with replacement of the red chromogen with a Fast Blue-based color development, Sigma, cat. F3378) for the anti-pERK antibody. Sections were not counterstained. The specificity of the pERK1/2 staining was controlled as indicated above.

Pictures of immunohistochemical stains were taken with the 100X/1.3, 40X/0.85, 20X/0.7 and 4/0.16 objectives (Olympus U Plan Apo) of an Olympus BX61 microscope equipped with an Olympus DP71 digital camera, using the Olympus cell^ $\mathrm{B}$ acquisition software.

\section{Western blotting}

Leukemic cells purified from the peripheral blood (as described $i^{5}$ ) of three patients with HCL were studied by Western blotting in parallel with BRAF-V600E analysis and pERK immunohistochemistry. Protein lysates in Laemmli sample buffer were separated by sodium dodecylsulfate-polyacrylamide gel electrophoresis on pre-cast electrophoresis gels (Any kD, Biorad, cat. 456-9036), transferred to a $0.45 \mu \mathrm{m}$-pore size nitrocellulose transfer membrane (Whatman GE Healthcare, cat. 10401196) and probed with anti-pERK1/2 antibody (1:2000 dilution, overnight at $\left.4^{\circ} \mathrm{C}\right)$, followed by membrane stripping (with Restore Western Blot Stripping Buffer, Pierce/Thermo scientific, cat. 21059) and re-probing with an antibody against total ERK1/2 (rabbit clone 137F5; Cell Signaling Technology, cat.4695; 1:1000 dilution, $2 \mathrm{~h}$ at room tem- perature). Upon incubation with a horseradish peroxidase-conjugated secondary donkey anti-rabbit antibody (Anti-Rabbit IgG, GE Healthcare Lifesciences, cat. NA934V; 1:8000 dilution, 45 min at room temperature), proteins were visualized using a chemiluminescence detection kit (Luminata Crescendo Western HRP Substrate, Millipore, cat. WBLUR0500). Protein lysates from either serum-starved ( 45 min at $37^{\circ} \mathrm{C}$ in RPMI1640) or unstarved human lymphoblastic T-cell line Jurkat cells were used to confirm the pERK origin of the Western blot bands by showing the expected decrease in the intensity of the pERK bands upon serum starvation.

\section{Results}

The BRAF-V600E mutation was detected by allele-specific PCR in all $37 \mathrm{HCL}$ cases (Table 1). Immunostaining of bone marrow biopsies showed pERK expression by HCL cells in 35/37 cases (Table 1 and Figure 1A). With the exception of $\mathrm{pERK} \mathrm{K}^{+}$endothelial cells and scattered stromal cells, pERK expression was restricted to leukemic cells, as indicated by correlating immunophenotype with morphology and also formally confirmed in 21 cases by double staining for pERK and the B-cell marker PAX5 (Figure 1B). The positivity was abolished by pre-incubation of the anti-pERK antibody with its blocking phospho-peptide in all 15 samples analyzed in this regard (Figure 1C), demonstrating the specificity of the staining. The staining pattern showed some variability among the HCL population. In fact, cytoplasmic-only positive hairy cells were most abundant but were occasionally admixed with HCL cells expressing pERK also in the nucleus (Figure 1D).

The most informative $\mathrm{pERK}$ immunostaining was usually observed in formalin-fixed samples antigen-retrieved at $97^{\circ} \mathrm{C}$, and documented pERK expression in virtually all leukemic cells (Figure 1A). In contrast, less intense and patchy positivity (often located at the periphery of the sample) was observed in biopsies antigen-retrieved at $85^{\circ} \mathrm{C}$ and/or fixed in B5 (Figure 2A-B). These results are not surprising since phospho-epitopes are known to be labile ${ }^{19}$ and can, therefore, be easily denatured by sample processing/fixation procedures. This may explain pERK negativity in two of 32 BRAF-V600E ${ }^{+}$HCL cases (Table 1), in which pERK expression was limited to a few faintly positive leukemic cells at the periphery of the section (with adjacent control stromal cells also being somewhat less strongly positive than in the other cases) (data not shown). Antigen denaturation in paraffin sections of these

Table 1. Phospho-ERK expression and BRAF-V600E mutation status in $\mathrm{HCL}$ and other B-cell leukemias/lymphomas.

\begin{tabular}{lcccc} 
& $\begin{array}{c}\text { pERK expression } \\
\text { cases } \\
\text { analyzed }\end{array}$ & $\begin{array}{c}\text { BRAF-VOGOE } \\
\text { positive }\end{array}$ & $\begin{array}{c}\text { cases } \\
\text { analyzed }\end{array}$ & $\begin{array}{c}\text { cases } \\
\text { positive }\end{array}$ \\
HCL & 37 & $35^{*}$ & 37 & 37 \\
SMZL & 40 & 0 & 40 & 0 \\
\hline HCL-variant & 2 & 0 & 1 & 0 \\
SLLU & 2 & 0 & 2 & 0 \\
\hline CD5- BLPD-NOS & 7 & 0 & 7 & 2
\end{tabular}

*Two HCL cases appeared negative on pERK immunohistochemistry likely due to denaturation of the phospho-epitope (see text). SLLU: splenic leukemia/lymphoma unclassifiable. 

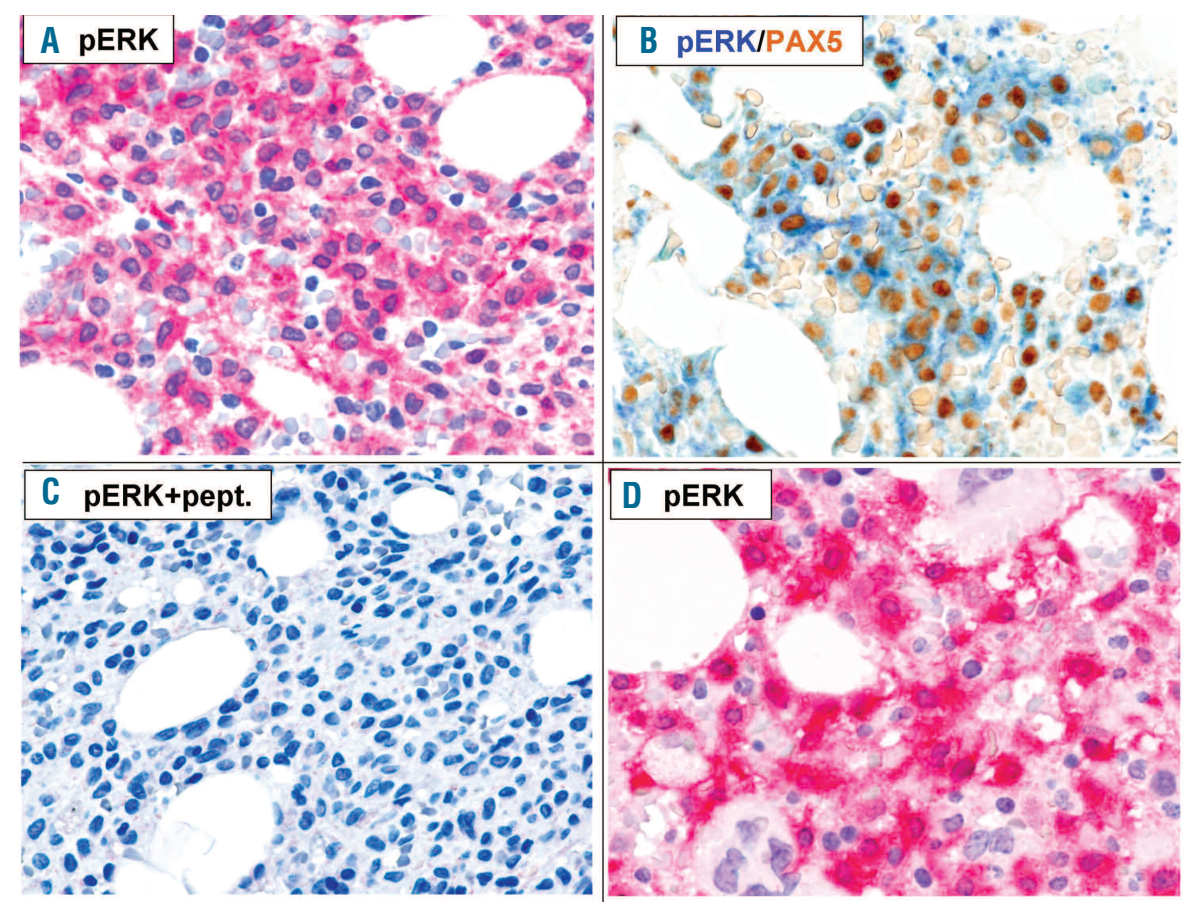

Figure 1. Phospho-ERK expression in HCL. A-C) Bone marrow sections of a HCL patient with diffuse infiltration by leukemic cells strongly expressing cytoplasmic pERK, as shown both in the single pERK staining $(A)$ and in the double staining of pERK (blue cytoplasmic positivity) and PAX5 (brown nuclear positivity) (B). The pERK staining is completely abolished $(\mathrm{C})$ by pre-incubation with the specific ERK phospho-peptide recognized by the anti-pERK antibody. (D) Leukemic hairy cells displaying pERK expression in the cytoplasm and the nucleus.
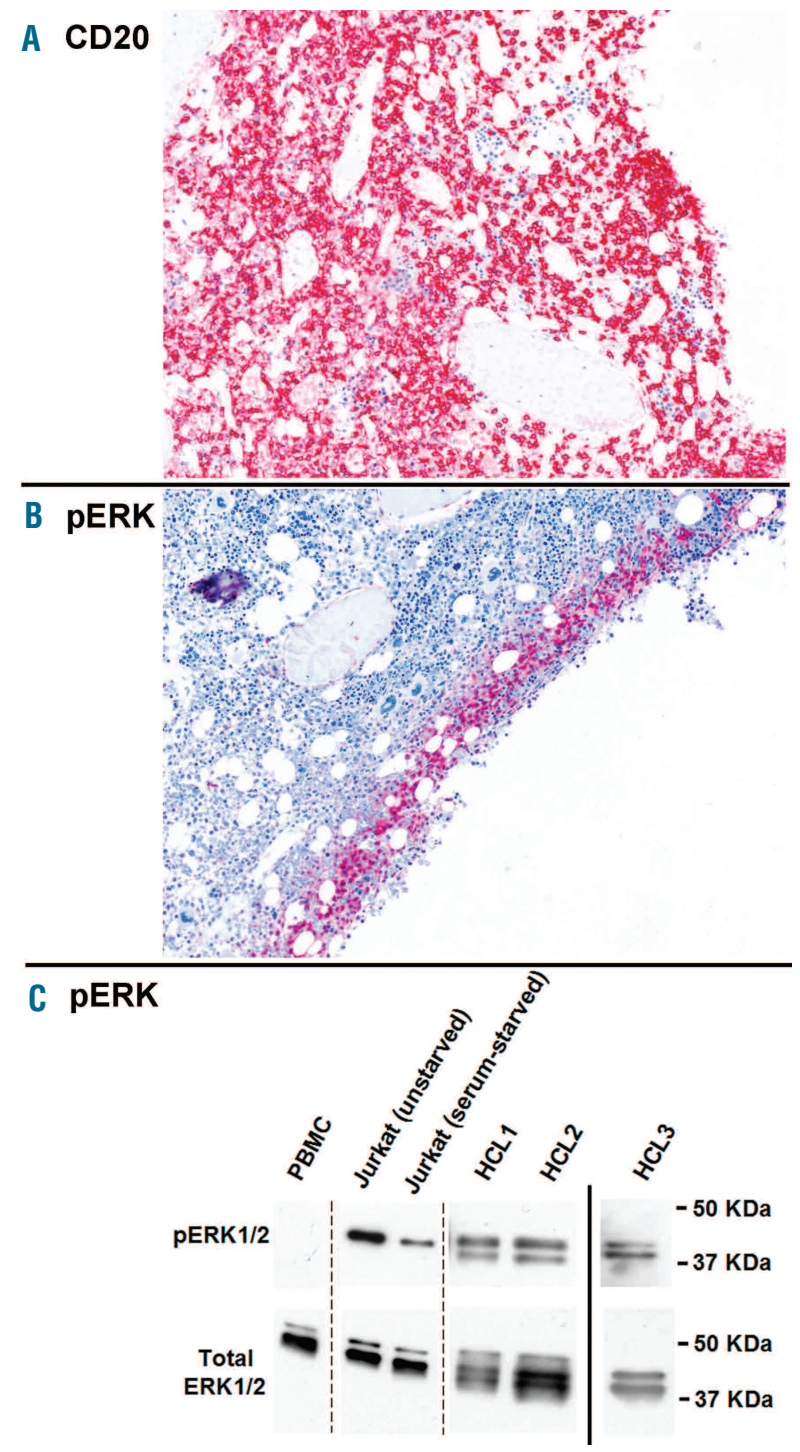

Figure 2. Antigenic denaturation of the phospho-ERK epitope. (A) B5fixed bone marrow section of a $\mathrm{HCL}$ patient with diffuse leukemic infiltration. Hairy cells show strong CD20 staining all over the section, whereas the pERK labeling is restricted to the most superficial few cell layers (B) due to antigen denaturation occurring deeper in the section (where also no control stromal cells are stained by the anti-pERK antibody). (C) Western blots showing (upper panels) expression of pERK1/2 (44/42 KDa molecular weight) in leukemic cells purified from the peripheral blood of three HCL patients (HCL1, HCL2 and HCL3, the last appearing largely negative on immunohistochemistry for pERK), but not in peripheral blood mononuclear cells of a healthy donor (PBMC). The phospho-ERK origin of the bands shown is confirmed by observing the expected decrease in intensity upon serum starvation of Jurkat T-lymphoblastic cells. The lower panels show Western blots of total ERK1/2 in the same samples as a loading control. Dashed lines indicate repositioned lanes of the same gel, whereas the solid line separates two different gels with comparable exposure times of the total ERK1/2 blot relative to the respective phospho-ERK1/2 blot.

cases was further supported by Western blotting studies that confirmed consistent pERK expression in all three BRAF-V600E ${ }^{+} \mathrm{HCL}$ patients for whom a sufficient number of purified HCL cells could be obtained (Figure 2C), including one of the two cases appearing largely negative on immunohistochemistry (the other one could not be analyzed by Western blotting).

Since the distinction between HCL and HCL-like disorders is clinically relevant, ${ }^{4}$ we next investigated whether these diseases differ in pERK expression by immunostaining bone marrow trephines or spleen specimens from 44 patients with HCL-like disorders and seven with BLPDNOS, in all of whom (except 1 with HCL-variant) the BRAF-V600E status could be investigated.

Allele-specific PCR showed the absence of BRAF-V600E in 43/43 HCL-like disorders and in 5/7 BLPD-NOS (Table 1). The remaining two cases of BLPD-NOS are extremely rare examples of non-HCL B-cell leukemias/lymphomas harboring BRAF-V600E.? On immunohistochemistry, all 44 HCL-like cases (Figure 3A-C) and all seven BLPD-NOS were negative for $\mathrm{pERK}$ (Table 1). Interestingly, in the two cases of BLPD-NOS harboring BRAF-V600E, pERK staining was strong in normal stromal cells but absent in adja- 

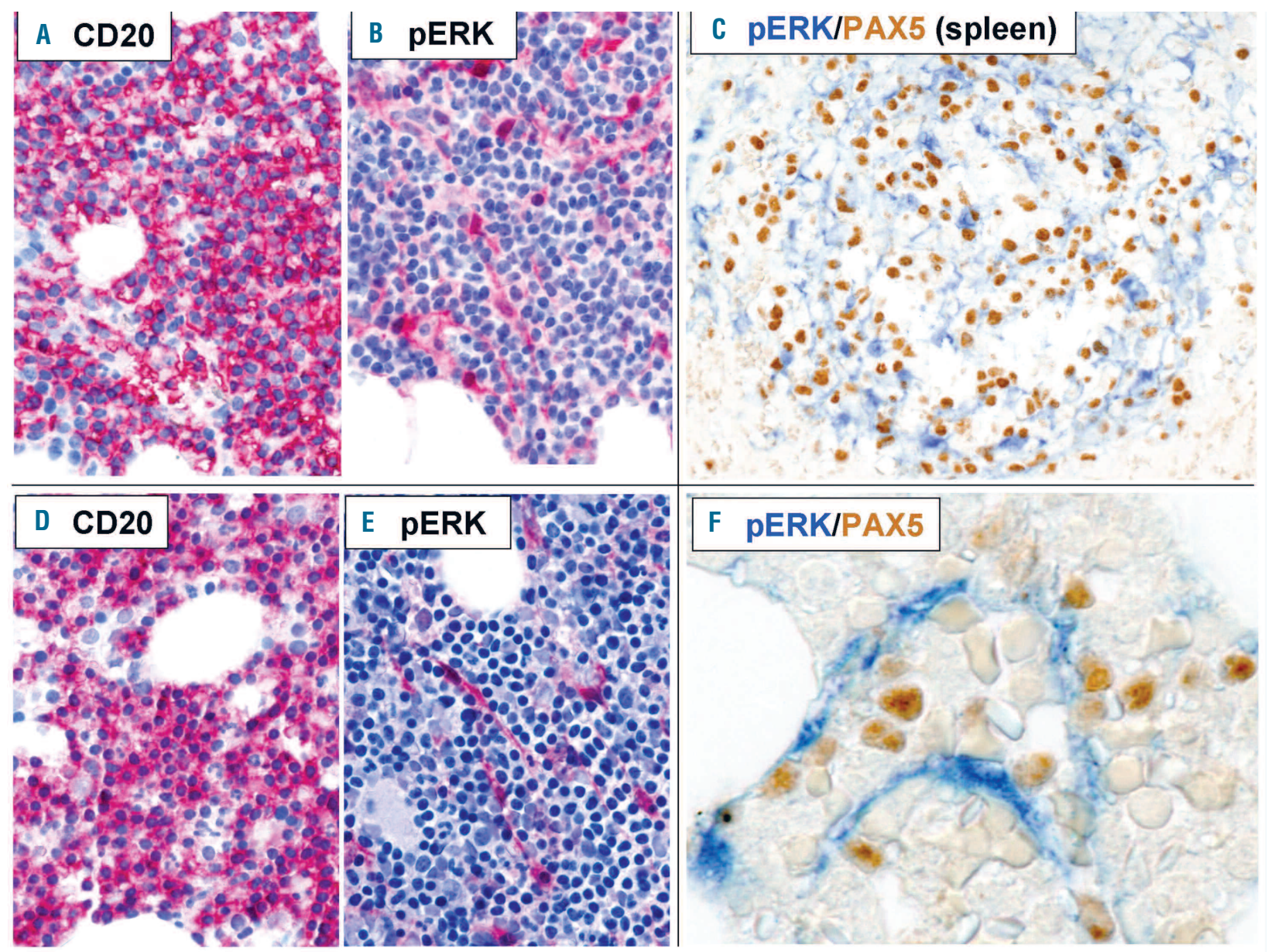

Figure 3. Lack of phospho-ERK expression in HCL-like leukemias/lymphomas. (A-B) Bone marrow sections of a patient with HCL-variant showing diffuse infiltration by leukemic cells expressing CD20 (A), but lacking expression of pERK (B). Stromal cells admixed with the leukemic cells show strong pERK expression (B) and serve as an internal positive control for the $p E R K$ staining. (C) Splenectomy specimen of a patient with SMZL showing a neoplastic white-pulp nodule, double stained for pERK and PAX5. Lymphoma cells, showing the PAX5 brown nuclear staining, do not express pERK, whereas adjacent control stromal cells do (blue cytoplasmic and nuclear staining). (D-E) Bone marrow sections of one of the two patients with BLPD-NOS harboring the BRAF-V600E mutation at allele-specific-PCR: ${ }^{7}$ marked infiltration by leukemic cells expressing $\mathrm{CD}^{2} \mathrm{O}^{+}(\mathrm{D})$ but not $\mathrm{pERK}(\mathrm{E})$. Admixed stromal cells strongly positive for pERK (E) serve as an internal positive control. (F) Bone marrow section of the other patient with BLPD-NOS harboring the BRAF-V600E mutation at allele-specific PCR. ${ }^{7}$ Double staining for pERK and PAX5 shows that leukemic B cells (PAX5 brown nuclear staining) are negative for pERK, which is instead strongly expressed by admixed control stromal cells (blue cytoplasmic and nuclear staining).

cent leukemic cells (Figure 3D-F), making antigen denaturation an unlikely cause of the undetectable pERK staining in the leukemic cells.

\section{Discussion}

By correlating the expression of pERK (as assessed by routine immunohistochemistry) to the BRAF-V600E mutation status (as assessed by allele-specific PCR) in a substantial number of HCL and HCL-like cases (37 and 44, respectively), this study provides solid evidence that the RAF-MEK-ERK pathway is aberrantly activated in the former as opposed to the latter.

Our results have implications for the diagnosis of HCL. BRAF-V600E detection by PCR is the gold standard for the genetic diagnosis of HCL. However, not all laboratories are equipped for molecular biology studies, especially in developing countries, whilst immunohistochemistry is widely available. Thus, immunohistological surrogates for the genetic diagnosis of HCL would be helpful. Phospho-ERK immunohistochemistry can indirectly document the presence of BRAF-V600E by looking at the activation of BRAF downstream targets. A potential limitation of this method may be that mechanisms other than BRAF-V600E can lead to ERK phosphorylation in any given cell type. However, in the context of the differential diagnosis between HCL and HCL-like neoplasms, we have observed a very close concordance between BRAF-V600E and ERK phosporylation status. Thus, pERK immunostaining may help (in addition to annexin-120 in distinguishing HCL from HCL-like disorders and, although we used immunohistochemistry to detect pERK expression, this staining is in principle amenable also to flow cytometry, another widely available tool.

Phospho-ERK immunohistochemistry was also instrumental in shedding light on the significance of the BRAFV600E mutation in two exceptionally rare examples of non-HCL B-cell leukemias/lymphomas previously shown to harbor this mutation by a sensitive allele-specific PCR assay. Indeed, the lack of pERK expression by leukemic 
cells in these two patients, together with the faint BRAFV600E bands produced by allele-specific PCR even in the case showing major leukemic cell infiltration of the bone marrow $(60 \%))^{7}$ suggest that BRAF-V600E ${ }^{+}$leukemic cells represent a minor subclone of these two BLPD-NOS or (perhaps less likely) define a small HCL clone subclinically co-existing with the BLPD-NOS as a separate neoplasm.

MEK-ERK pathway activation had been previously reported to sustain leukemic cell survival in a few HCL patients, ${ }^{15}$ although the mechanism of activation was believed to be BRAF-independent and was not conclusively clarified. The results presented here demonstrate in a substantial number of HCL patients a constant association of BRAF-V600E with ERK phosphorylation. These findings and our previous report that MEK and ERK are dephosphorylated upon in vitro exposure of $\mathrm{HCL}$ cells to a BRAF inhibitor ${ }^{5}$ strongly support the clinical use of specific BRAF and/or MEK inhibitors ${ }^{16-18}$ to block RAF-MEK-ERK activity in HCL patients. It is also anticipated that pERK immunostaining of bone marrow biopsies taken at different time points during treatment with BRAF/MEK inhibitors may serve as a biomarker of response to therapy. In addition, this technique has the potential for detect- ing the emergence of $\mathrm{pERK}^{+} \mathrm{HCL}$ subclones that may become resistant to these inhibitors through MEK-ERK pathway re-activation via alternative mechanisms, as observed in patients with melanoma. ${ }^{21}$

\section{Funding}

This work was supported by the Associazione Italiana Ricerca Cancro (grants IG10470 and MCO 10007), Ministero dell'Istruzione-Università-Ricerca (MIUR; Progetto Futuro in Ricerca 2010 - RBFR10WT2K), Ministero della Salute (Progetto Giovani Ricercatori GR-2010-2303444), Hairy Cell Leukemia Research Foundation and Fondazione Cassa di Risparmio di Perugia.

\section{Acknowledgments}

We are grateful to Barbara Bigerna and Alessandra Pucciarini for technical help, and to Claudia Tibidò for secretarial assistance.

\section{Authorship and Disclosures}

Information on authorship, contributions, and financial \& other disclosures was provided by the authors and is available with the online version of this article at www. haematologica.org.

\section{References}

1. Foucar K., Falini B, Catovsky D, Stein H. Hairy cell leukaemia. In: Swerdlow S, Campo E, Harris NL, et al., ed. WHO Classification of Tumours of Haematopoietic and Lymphoid Tissues (4th ed.). Lyon: International Agency for Research on Cancer (IARC); 2008:188-90.

2. Basso K, Liso A, Tiacci E, Benedetti R, Pulsoni A, Foa R, et al. Gene expression profiling of hairy cell leukemia reveals a phenotype related to memory B cells with altered expression of chemokine and adhesion receptors. J Exp Med. 2004;199(1):5968.

3. Tiacci E, Liso A, Piris M, Falini B. Evolving concepts in the pathogenesis of hairy-cell leukaemia. Nat Rev Cancer. 2006;6(6):43748.

4. Grever MR. How I treat hairy cell leukemia. Blood. 2010;115(1):21-8.

5. Tiacci E, Trifonov V, Schiavoni G, Homes A, Kern W, Martelli MP, et al. BRAF mutations in hairy-cell leukemia. N Engl J Med. 2011;364(24):2305-15

6. Tiacci E, Schiavoni G, Forconi F, Santi A, Trentin L, Ambrosetti A, et al. Simple genetic diagnosis of hairy cell leukemia by sensitive detection of the BRAF-V600E mutation. Blood. 2012;119(1):192-5.

7. Arcaini L, Zibellini S, Boveri E, Riboni R, Rattotti S, Varettoni M, et al. The BRAF V600E mutation in hairy cell leukemia and other mature B-cell neoplasms. Blood.
2012;119(1):188-91

8. Boyd EM, Bench AJ, van't Veer MB, Wright P, Blosham DM, Follows CA, et al. High resolution melting analysis for detection of BRAF exon 15 mutations in hairy cell leukaemia and other lymphoid malignancies. Br J Haematol. 2011;155(5):609-12.

9. Blombery PA, Wong SQ, Hewitt CA Dobrovic A, Maxwell EL, Juneja S, et al. Detection of BRAF mutations in patients with hairy cell leukemia and related lymphoproliferative disorders. Haematologica. 2012;97(5):780-3.

10. Lennerz JK, Klaus BM, Marienfeld RB, Moller P. Pyrosequencing of BRAF V600E in routine samples of hairy cell leukaemia identifies CD5+ variant hairy cell leukaemia that lacks V600E. Br J Haematol. 2012;157(2):267-9.

11. Schnittger $S$, Bacher $U$, Haferlach $T$ Wendland N, Ulke M, Dicker F, et al. Development and validation of a real-time quantification assay to detect and monitor BRAFV600E mutations in hairy cell leukemia. Blood. 2012;119(13):3151-4.

12. Keshet Y, Seger R. The MAP kinase signaling cascades: a system of hundreds of components regulates a diverse array of physiological functions. Methods Mol Biol. 2010;661:3-38.

13. Davies H, Bignell GR, Cox C, Stephens P, Edkins S, Clegg S, et al. Mutations of the BRAF gene in human cancer. Nature. 2002;417(6892):949-54

14. Wan PT, Garnett MJ, Roe SM, Lee S, Niculescu-Duvaz D, Good VM, et al.
Mechanism of activation of the RAF-ERK signaling pathway by oncogenic mutations of B-RAF. Cell. 2004;116(6):855-67.

15. Kamiguti AS, Harris RJ, Slupsky JR, Baker PK, Cawley JC, Zuzel M. Regulation of hairy-cell survival through constitutive activation of mitogen-activated protein kinase pathways. Oncogene. 2003;22(15): 2272 84.

16. Chapman PB, Hauschild A, Robert C, Haanen JB, Ascierto P, Larkin J, et al. Improved survival with vemurafenib in melanoma with BRAF V600E mutation. N Engl J Med. 2011;364(26):2507-16.

17. Flaherty KT, Robert C, Hersey P, Nathan P, Garbe C, Milhem M, et al. Improved survival with MEK inhibition in BRAF-mutated melanoma. N Engl J Med. 2012;367(2): 107-14.

18. Hauschild A, Grob JJ, Demidov LV, et al. Dabrafenib in BRAF-mutated metastatic melanoma: a multicentre, open-label, phase 3 randomised controlled trial. Lancet. 2012;380(9839):358-65.

19. Mandell JW. Phosphorylation state-specific antibodies: applications in investigative and diagnostic pathology. Am J Pathol. 2003;163(5):1687-98.

20. Falini B, Tiacci E, Liso A, Basso K, Sabattini E, Pacini R, et al. Simple diagnostic assay for hairy cell leukaemia by immunocytochemical detection of annexin A1 (ANXA1). Lancet. 2004;363(9424):1869-70.

21. Solit DB, Rosen N. Resistance to BRAF inhibition in melanomas. $\mathrm{N}$ Engl J Med. 2011;364(8):772-4. 